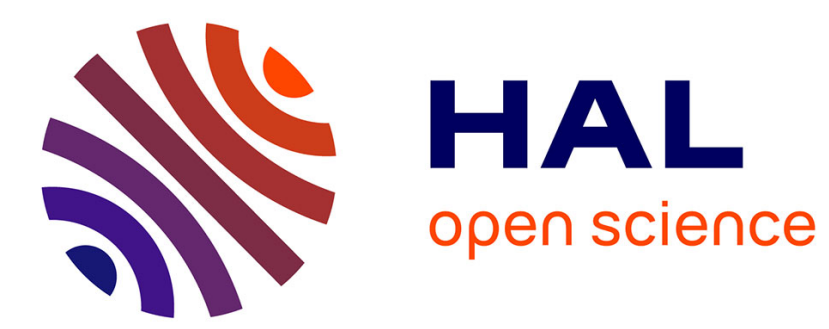

\title{
Homogeneous Auxin Steady States and Spontaneous Oscillations in Flux-Based Auxin Transport Models
}

\author{
Etienne Farcot, Yuan Yuan
}

\section{To cite this version:}

Etienne Farcot, Yuan Yuan. Homogeneous Auxin Steady States and Spontaneous Oscillations in Flux-Based Auxin Transport Models. SIAM Journal on Applied Dynamical Systems, 2013, 12 (3), pp.1330-1353. 10.1137/120891538 . hal-00850927

\section{HAL Id: hal-00850927 https://hal.inria.fr/hal-00850927}

Submitted on 9 Aug 2013

HAL is a multi-disciplinary open access archive for the deposit and dissemination of scientific research documents, whether they are published or not. The documents may come from teaching and research institutions in France or abroad, or from public or private research centers.
L'archive ouverte pluridisciplinaire HAL, est destinée au dépôt et à la diffusion de documents scientifiques de niveau recherche, publiés ou non, émanant des établissements d'enseignement et de recherche français ou étrangers, des laboratoires publics ou privés. 


\title{
Homogeneous Auxin Steady States and Spontaneous Oscillations in Flux-Based Auxin Transport Models*
}

\author{
Etienne Farcot $^{\dagger}$ and Yuan Yuan ${ }^{\ddagger}$
}

\begin{abstract}
We consider patterns formed by active transport of auxin in a tissue, where the accumulation of transporters is activated by local fluxes of auxin. We characterize the steady states for which auxin is homogeneous in the tissue. Under a condition of regularity of the dependence of transporters to the flux, we can prove that one of these steady states, with zero flux everywhere, is always locally asymptotically stable. When the condition of regularity is not satisfied, by a combination of analytic and numeric results, we show that the same steady state may undergo bifurcations and become unstable. In particular, we can observe stable oscillations via Hopf bifurcation in the system having the form of a row of cells. This paper presents that flux-based active transport alone is enough to induce spontaneous oscillations of auxin in a tissue.
\end{abstract}

Key words. active transport, auxin, bifurcations

AMS subject classifications. $34 \mathrm{C} 23,34 \mathrm{C} 60,92 \mathrm{C} 15,92 \mathrm{C} 80$

DOI. $10.1137 / 120891538$

1. Introduction. Auxin is a major plant hormone, involved in a large number of physiological processes, and is known to be distributed in a complex way in tissues. Although auxin can enter cells by free diffusion, the $\mathrm{pH}$ difference between plant cells and the intercellular matrix precludes auxin from leaving cells without the intermediate active efflux transporters distributed on cell membranes. Among the most studied of these transporters are the so-called PIN proteins, which are distributed in a polar way on cell membranes, through a mechanism that is not entirely known to date. It has been observed that this polar intracellular distribution is correlated with local differences in auxin concentration. Based on these observations, it is commonly assumed that there is a causal relationship and that PIN polarization is a function of auxin distribution among neighboring cells. This general assumption has led to several mathematical formulations in the literature (reviewed, e.g., in [14]), whose differences reflect the still incomplete mechanistic knowledge of the process of PIN distribution. These formulations can be broadly classified in two main classes:

- Concentration-based models: Assume that PIN proteins accumulate on cell membranes

\footnotetext{
* Received by the editors September 17, 2012; accepted for publication (in revised form) by R. Albert May 10, 2013; published electronically July 30, 2013.

http://www.siam.org/journals/siads/12-3/89153.html

†School of Mathematical Sciences, University of Nottingham, University Park, Nottingham, NG7 2RD, United Kingdom, and Centre for Plant Integrative Biology, University of Nottingham, Sutton Bonington LE12 5RD, United Kingdom (etienne.farcot@nottingham.ac.uk). Previously at Virtual Plants Inria team, C.C. 06002, 34095 Montpellier, France (etienne.farcot@inria.fr). The research of this author was partially supported by the "Explorateur" program, a mobility program of the Department of International Affairs, Inria, France.

¥Department of Mathematics and Statistics, Memorial University of Newfoundland, St. John’s, NL A1C 5S7, Canada (yyuan@mun.ca). The research of this author was partially supported by the Natural Sciences and Engineering Research Council of Canada.
} 
with a rate that depends on the local differences of auxin concentration or in fact the relative levels of auxin between nearby cells.

- Flux-based models: Assume that PIN accumulates on cell membranes as a function of the flux of auxin through them.

These models have been initially introduced for distinct purposes: flux-based models were supposed to account for the vein-like patterns observed notably in leaves $[19,20,21]$, whereas concentration-based models have been introduced more recently to account for the regular, spot-like, patterns of phyllotaxis (the regular arrangement of plant organs at the shoot apex) [13, 26]. However, some recent studies have indicated that both types of models could in fact generate a broader range of patterns than initially thought. To mention some examples, computer simulations based on representations of realistic tissues at a cellular resolution have reproduced realistic patterns of phyllotaxis, both with concentration-based $[13,26]$ and flux-based [27] models. Likewise, vein-like patterns have been observed in both concentration-based [15] and flux-based [10] models. The mathematical analysis in [25] has shown that concentration-based models were able to generate various patterns, including both stripes and spots. In principle, this could account respectively for phyllotactic and venation patterns. Alternatively, a composite model including both flux-based and concentration-based regulation has also been proposed to account simultaneously for several types of patterns [2].

Based on these studies, it is not possible to clearly decide whether one of the two classes of models discussed above has more biological validity than the other, since both types of models reproduce both main types of known auxin patterns, at least qualitatively. Nor is there any clear experimental evidence to support one or the other of the two classes of auxin transport models - a fact that currently leads to some debate among scientists. One way to contribute to this debate would be to determine some qualitative property that is satisfied by one of the two classes of models and not by the other. The strategy in this paper is to start by analytically studying the ability for pattern generation in flux-based models, which can be compared with the case of concentration-based models which have been studied mathematically in $[8,9,25]$.

A number of previous works have considered auxin distribution in tissues which already contained some prepattern, usually taking the form of local sources or sinks of auxin; see, e.g., [10]. However, more mathematical studies usually do not assume prepatterns and start by considering steady states with homogeneous auxin; see, e.g., [8, 9, 25]. The underlying goal, following the seminal work of Turing on reaction-diffusion systems [28], is to consider whether patterns may emerge from a nonpatterned situation, i.e., a steady state with homogeneous auxin distribution, under conditions on parameters for which this "no-pattern" situation becomes unstable, leading to the formation of actual patterns. Using a similar strategy allows us to illustrate a striking specificity of flux-based models: several configurations of PIN transporters may lead to the same distribution of auxin at steady state, in particular a homogeneous distribution. When one steady state with homogeneous auxin becomes unstable, one may expect to observe alternative stable steady states, which can be either uniform in auxin with a different PIN configuration or nonuniform in auxin. Because of this multiplicity of homogeneous auxin patterns, we put a particular focus on uniform auxin steady states and their stability.

We consider typical flux-based models and concentrate on the analytic study, from the point of view of dynamical systems with the help of graphical simplification. The strategy

Copyright $\odot$ by SIAM. Unauthorized reproduction of this article is prohibited. 
undertaken in the present paper is complementary to the numerical simulation studies: we choose to include only the most basic features compatible with a given transport hypothesis, resulting in a relatively small number of parameters. This allows us to begin a systematic exploration of the possible transport patterns. One underlying goal is to determine some qualitative features at the cellular level in terms of tissue scale patterns, where experimental observation is easier.

In the following section, a generic mathematical formulation of flux-based models is presented. Then, in section 3, steady states with uniform auxin distribution are characterized, with some additional results on their stability, for both arbitrary and regular tissues, with a focus on ring-shaped structures. Numerical results on some dynamical behavior exhibited on a tissue taking the form of a ring or row of cells are presented in section 4 , followed by a conclusion and discussion on biological implications and future work.

2. Model formulation. In this section we consider the active transport of auxin in a tissue with a time span that is typically shorter than cell division (which occurs approximately at a period of 12 to $24 \mathrm{~h}$ ), so that the number of cells is fixed, denoted $N$. Each cell $i$ has a set $\mathcal{N}_{i} \subset\{1 \ldots N\}$ of neighbors. We denote by $V_{i}\left(m^{3}\right)$ the volume of cell $i$ and by $A_{i j}\left(m^{2}\right)$ the exchange surface area between cell $i$ and $j$. If cells $i$ and $j$ are not neighbors, one has $A_{i j}=0$ and, in particular, $A_{i i}=0$. Obviously, $A_{i j}=A_{j i}$, and thus the matrix $A=\left(A_{i j}\right)$ is symmetric and nonnegative, with zero diagonal elements.

To describe the dynamics of auxin transport in a tissue, we use $a_{i}\left(\mathrm{~mol} \cdot \mathrm{m}^{-3}\right)$ to present the concentration of auxin in a cell $i$ and $P_{i j}\left(\mathrm{~mol} \cdot \mathrm{m}^{-2}\right)$ to present the concentration of transporter proteins (PIN) in the membrane facilitating transport from cell $i$ to cell $j$. In general, $P_{i j}$ and $P_{j i}$ may be different. The variation of auxin $a_{i}$ in cell $i$ is due to four processes:

- local synthesis, with a constant rate $\kappa\left(\mathrm{mol} \cdot \mathrm{m}^{-3} \cdot \mathrm{s}^{-1}\right)$ at which auxin is produced in cells,

- decay, with a rate $\delta\left(s^{-1}\right)$,

- free diffusion toward neighboring cells $j \in \mathcal{N}_{i}$, modeled by $D\left(a_{i}-a_{j}\right)\left(\mathrm{mol} \cdot \mathrm{m}^{-2} \cdot \mathrm{s}^{-1}\right)$ according to Fick's first law, where $D\left(\mathrm{~m} \cdot \mathrm{s}^{-1}\right)$ is the diffusion coefficient, and

- active transport by the transporter (PIN) proteins, modeled by $T\left(P_{i j} a_{i}-P_{j i} a_{j}\right)(\mathrm{mol}$. $\left.m^{-2} \cdot s^{-1}\right)$, adopting from [21], where $T\left(m^{3} \cdot \mathrm{mol}^{-1} \cdot \mathrm{s}^{-1}\right)$ is a transport efficiency coefficient.

The combination of the last two processes is called the flux of auxin from cell $i$ to cell $j$ and is supposed to induce the insertion of PIN proteins $P_{i j}$. We denote the flux as

$$
\Phi_{i j}=\left(T P_{i j}+D\right) a_{i}-\left(T P_{j i}+D\right) a_{j}
$$

This expression of flux was first derived in [19] for an abstract transporter substance and was reformulated more recently in terms of PIN protein concentrations $[10,13,24]$. The flux from cell $j$ to cell $i$ is thus the opposite: $\Phi_{j i}=-\Phi_{i j}$. The intensity of PIN insertion due to the feedback of the auxin flux is described by a continuous, increasing function $h: \mathbb{R} \rightarrow \mathbb{R}_{+}$. Since the active transport is directional, when more auxin comes in than goes out, no additional PIN is inserted. Based on this remark we make the assumption that, as in [19] and more 
recent studies [10, 24, 27],

$$
h\left(\Phi_{i j}\right)=0 \text { whenever } \Phi_{i j} \leq 0 \text { and } h\left(\Phi_{i j}\right)>0 \text { for } \Phi_{i j}>0 .
$$

With homogeneous auxin, Figure 1 shows the auxin and transporter proteins in two neighboring cells. We use the following graphical convention in all of our figures: the absence of auxin in a cell (resp., transporter proteins) is represented by white, while the highest concentration is shown in bright green (resp., red). Intermediate concentrations are represented by interpolations between these two extremes.

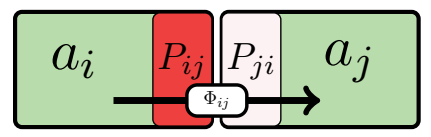

Figure 1. Two neighboring cells with concentrations of auxin and transporter proteins, and flux from $i$ to $j$.

The variation of concentration of PIN proteins $P_{i j}$ includes two processes:

- background insertion and removal of PIN, with an insertion rate $\rho_{0}\left(\mathrm{~mol} \cdot \mathrm{m}^{-2} \cdot \mathrm{s}^{-1}\right)$ and a removal rate $\mu\left(s^{-1}\right)$, and

- insertion induced by the flux $h\left(\Phi_{i j}\right)$.

Therefore the dynamics of auxin and transporter proteins in each cell $i \in\{1, \ldots, N\}$ can be described by the following system of coupled ordinary differential equations (where $j \in \mathcal{N}_{i}$ is any neighbor of cell $i$ ):

$$
\begin{aligned}
\frac{d a_{i}}{d t} & =\frac{1}{V_{i}} \sum_{j=1}^{N} A_{i j} \Phi_{j i}+\kappa-\delta a_{i}, \\
\frac{d P_{i j}}{d t} & =h\left(\Phi_{i j}\right)+\rho_{0}-\mu P_{i j} .
\end{aligned}
$$

Typical choices of $h$, as found in the literature [10, 27], can be (for positive $\Phi$ )

$$
\begin{gathered}
h_{1}(\Phi)=\rho \frac{\Phi^{n}}{\theta^{n}+\Phi^{n}} \text { with saturating coefficient } \theta \text { or } \\
h_{2}(\Phi)=\rho \Phi^{n} \text { without saturation, }
\end{gathered}
$$

where $\rho$ is a scaling coefficient. From the definition of $h$ in (2.1), we know that the left derivative at zero, $h^{\prime}\left(0^{-}\right)=\lim _{\varepsilon \rightarrow 0} \frac{h(-\varepsilon)}{\varepsilon}=0$, always holds for all $n$, while the right derivative $h_{i}^{\prime}\left(0^{+}\right)=\lim _{\varepsilon \rightarrow 0} \frac{h(\varepsilon)}{\varepsilon}=0(i=1,2)$ when $n>1$ and $h_{1}^{\prime}\left(0^{+}\right)=\frac{\rho}{\theta}, h_{2}^{\prime}\left(0^{+}\right)=\rho$ when $n=1$, implying that $h_{i}^{\prime}(0)$ is always zero when $n>1$ and does not exist when $n=1$; in other words, $h_{i}^{\prime}(0)$ is discontinuous with $h_{i}^{\prime}\left(0^{+}\right)>0$ and $h_{i}^{\prime}\left(0^{-}\right)=0$ for $i=1,2$ and $n=1$. From a general point of view, this discontinuity of the derivative is acceptable in some real applications, although the mathematical analysis is more difficult. Unless specified explicitly, we simply assume that $h$ is continuous and monotone increasing and that it satisfies (2.1).

Copyright $\odot$ by SIAM. Unauthorized reproduction of this article is prohibited. 
Before any further analysis, by rescaling the variables and parameters as

$$
\widetilde{P_{i j}}=T\left(P_{i j}-\frac{\rho_{0}}{\mu}\right), \quad \widetilde{D}=D+\frac{\rho_{0} T}{\mu}
$$

and omitting the tildes for simplicity, we will work on the following equations:

$$
\begin{aligned}
\Phi_{i j} & =\left(P_{i j}+D\right) a_{i}-\left(P_{j i}+D\right) a_{j}, \\
\frac{d a_{i}}{d t} & =\frac{1}{V_{i}} \sum_{j=1}^{N} A_{i j} \Phi_{j i}+\kappa-\delta a_{i}, \\
\frac{d P_{i j}}{d t} & =h\left(\Phi_{i j}\right)-\mu P_{i j} .
\end{aligned}
$$

It is convenient to represent cells and their neighboring relations as a graph. We denote this graph by $\mathcal{G}=(\mathcal{V}, \mathcal{E})$, with $\mathcal{V}=\{1, \ldots, N\}$, and $\mathcal{E} \subset \mathcal{V} \times \mathcal{V}$ is such that $(i, j) \in \mathcal{E}$ if and only if $A_{i j} \neq 0$. The set of neighbors of cell $i$ is thus characterized by $\mathcal{N}_{i}=\{j:(i, j) \in \mathcal{E}\}$. Furthermore, the flux of auxin between neighboring cells defines an orientation of the graph $\mathcal{G}$ : for each pair of neighbors $(i, j) \in \mathcal{E}$ one considers the orientation $i \rightarrow j$ if $\Phi_{i j} \geq 0$. The corresponding directed graph will be denoted by $\mathcal{G}^{+}(\Phi)$ hereafter. Based on Figure 1, a sample of a ring of $N$ cells is given in Figure 2(a). When the auxin in all cells is homogeneous, the associated oriented graph $\mathcal{G}^{+}(\Phi)$ with positive fluxes is shown in Figure 2(b).

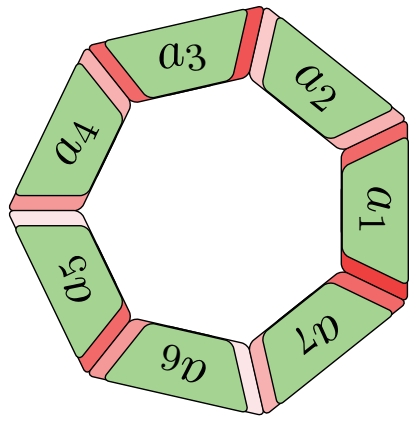

(a)

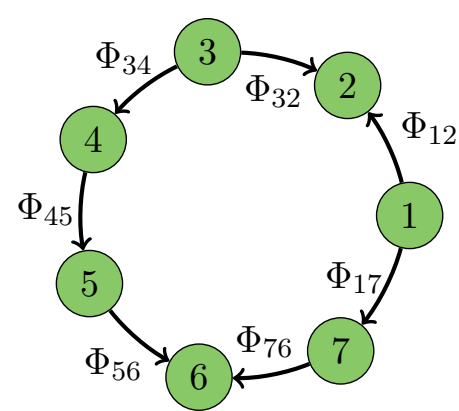

(b)

Figure 2. (a) A ring of $N(N=7)$ cells, with components shaded according to the concentration of transporter proteins. (b) The associated oriented graph $\mathcal{G}^{+}(\Phi)$ with positive fluxes.

Recall that only terms in (2.3)-(2.4) corresponding to neighbor cells are nonzero. We denote the cardinality of neighbor sets by $d_{i}=\operatorname{card}\left(\mathcal{N}_{i}\right)$ and call it the degree of cell $i$. Then $d=\sum_{i=1}^{N} d_{i}$ is the total degree of the graph $\mathcal{G}$, and the system $(2.3)-(2.4)$ consists of $N+d$ equations.

It is important to note that in the model (2.3)-(2.4) the dynamics of $P_{i j}$ does not directly depend on the amount of PIN allocated to other sides of the same cell. However, some experimental evidence suggests that the amount of PIN available in each cell is fixed, or slowly varying [11], and it was shown in [10] that taking this effect into account had a noticeable effect on patterns of auxin observed in numerical simulations. 
To assess the consequences of our assumption, let us consider an alternative model, where $a_{i}$ evolves as in (2.3), but with different PIN dynamics. We use additional variables $P_{i}$ representing the concentration of PIN in cell $i$. Suppose that PIN is produced and degraded with rates $\varepsilon \rho_{P}$ and $\varepsilon \delta_{P}$, respectively, where the coefficient $\varepsilon$ indicates that these processes are slow. PIN is then reallocated among sides with a rate that is proportional to its concentration $P_{i}$ and to the function $h$ of auxin flux, with a transfer rate denoted $\lambda$. The removal rate $\mu$ is as in (2.4):

$$
\begin{aligned}
\frac{d P_{i}}{d t} & =\varepsilon\left(\rho_{P}-\delta_{P} P_{i}\right)+\sum_{j \in \mathcal{N}_{i}}\left(\mu P_{i j}-\lambda P_{i} h\left(\Phi_{i j}\right)\right), \\
\frac{d P_{i j}}{d t} & =\lambda P_{i} h\left(\Phi_{i j}\right)-\mu P_{i j} .
\end{aligned}
$$

The model considered in [10] is equivalent to the form obtained by taking the limit $\varepsilon \rightarrow 0$ of infinitely slow production and decay of PIN. The steady states for the two equations (2.5)-(2.6) are given by

$$
P_{i}=\frac{\rho_{P}}{\delta_{P}} \quad \text { for all } i, \text { regardless of } \varepsilon, \text { and } P_{i j}=\frac{\lambda \rho_{P}}{\mu \delta_{P}} h\left(\Phi_{i j}\right) .
$$

It appears that the last equation above differs from the steady state for (2.4) only by a factor $\frac{\lambda \rho_{P}}{\delta_{P}}$. In other words, all the possible steady states of the model $(2.3),(2.5)-(2.6)$ including PIN reallocation can be found through the study of the simpler model (2.3)-(2.4), on which we base the rest of this work.

3. Homogeneous auxin steady states and their stability. In this section we focus on steady states for which the auxin levels in a tissue are identical in every cell. We first present some results for a tissue with arbitrary structure and then provide further analysis for a regular tissue.

3.1. Arbitrary tissue structure. From (2.3)-(2.4), a steady state $\left(a_{i}^{*}, P_{i j}^{*}\right)_{i, j}$ satisfies the following for all $i, j$ :

$$
\left\{\begin{array}{l}
a_{i}^{*}=\frac{1}{\delta V_{i}} \sum_{j=1}^{N} A_{i j} \Phi_{j i}^{*}+\frac{\kappa}{\delta}, \\
P_{i j}^{*}=\frac{1}{\mu} h\left(\Phi_{i j}^{*}\right), \quad \text { with } \quad \Phi_{i j}^{*}=\left(P_{i j}^{*}+D\right) a_{i}^{*}-\left(P_{j i}^{*}+D\right) a_{j}^{*} .
\end{array}\right.
$$

Because the flux may induce the transporter proteins only when it is positive, any pair $\left(P_{i j}^{*}, P_{j i}^{*}\right)$ will satisfy certain constraints. Actually, from $\Phi_{i j}^{*}=-\Phi_{j i}^{*}$ it follows that one of the two terms $h\left(\Phi_{i j}^{*}\right), h\left(\Phi_{j i}^{*}\right)$ is always zero. Therefore, the following proposition holds.

Proposition 3.1. At steady state, for any $(i, j) \in \mathcal{E}$, at least one of the two values $P_{i j}^{*}, P_{j i}^{*}$ must be zero.

Suppose, for instance, that $\Phi_{j i}^{*} \leq 0$; then, the steady-state equations for $P_{j i}^{*}$ yield $P_{j i}^{*}=0$, and hence $\Phi_{i j}^{*}=P_{i j}^{*} a_{i}^{*}+D\left(a_{i}^{*}-a_{j}^{*}\right)$. For a fixed pair of steady-state values $\left(a_{i}^{*}, a_{j}^{*}\right)$, the steady state of $P_{i j}^{*}$ satisfies

$$
P_{i j}^{*}=\frac{1}{\mu} h\left(a_{i}^{*} P_{i j}^{*}+D\left(a_{i}^{*}-a_{j}^{*}\right)\right) .
$$

Copyright $\odot$ by SIAM. Unauthorized reproduction of this article is prohibited. 
From the hypotheses on $h$, whenever $a_{i}^{*} \leq a_{j}^{*}$, (3.2) admits a solution at $P_{i j}^{*}=0$. In particular, for homogeneous auxin $a_{i}^{*}=a^{*}$ for all $i$, we obtain the fixed point equation,

$$
P_{i j}^{*}=\frac{1}{\mu} h\left(a^{*} P_{i j}^{*}\right),
$$

which may in general have any number of positive solutions (including none). In the following, we will always assume that (3.3) admits a finite number $m+1$ (with $m \geqslant 0$ ) of nonnegative solutions and denote them by

$$
\mathcal{P}^{*}=\left\{\mathcal{P}_{0}^{*}, \mathcal{P}_{1}^{*}, \ldots, \mathcal{P}_{m}^{*}\right\},
$$

where $\mathcal{P}_{0}^{*}=0$ and all other $\mathcal{P}_{i}^{*}>0$. The rest of this section is devoted to characterizing the homogeneous steady states with $a_{i}^{*}=a^{*}$ for all $i$. Note that the value of $\mathcal{P}_{i}^{*}$ in (3.4) depends on $a^{*}$ in general.

Equation (3.3) and Proposition 3.1 constrain the value of $P_{i j}^{*}$ at a steady state, though do not completely determine it. The auxin concentration $a^{*}$, on the other hand, can take only one value if it is assumed to be homogeneous in a tissue.

Proposition 3.2. Suppose that there exists a steady state with homogeneous auxin, i.e., such that $a_{i}^{*}$ does not depend on $i$. Then, $a_{i}^{*}=a^{*}=\frac{\kappa}{\delta}$.

Proof. Assume $a_{i}^{*}=a^{*}$ for all $i \in\{1, \ldots, N\}$ at a steady state. Then, summing the first equation in (3.1) over $i$, one gets

$$
\sum_{i=1}^{N} \sum_{j=1}^{N} A_{i j} \Phi_{j i}^{*}=\left(\delta a^{*}-\kappa\right) \sum_{i=1}^{N} V_{i} .
$$

Since $A_{i j}=A_{j i}$ and $\Phi_{i j}^{*}=-\Phi_{j i}^{*}$, the left-hand side above must be zero; therefore, the claim follows due to $V_{i}>0$ for each $i$.

One consequence of Proposition 3.2 is that the set $\mathcal{P}^{*}$ in (3.4) can be defined using only the parameters of (2.3)-(2.4), since (3.3) can now be given a more specific form: $P_{i j}^{*}=$ $\frac{1}{\mu} h\left(a^{*} P_{i j}^{*}\right)=\frac{1}{\mu} h\left(\frac{\kappa}{\delta} P_{i j}^{*}\right)$. Define the scalar function

$$
\begin{aligned}
\widehat{h}: \mathbb{R}_{+} & \longrightarrow \mathbb{R}_{+}, \\
\xi & \longmapsto \widehat{h}(\xi)=\frac{1}{\mu} h\left(\frac{\kappa}{\delta} \xi\right) .
\end{aligned}
$$

Then, with homogeneous auxin, the steady-state solutions for $P_{i j}^{*}$ will be determined by the nonnegative fixed points of the function $\widehat{h}$. This provides us with a definition for the set $\mathcal{P}^{*}$ :

$$
\mathcal{P}^{*}=\operatorname{Fix}(\widehat{h})=\{\xi: \widehat{h}(\xi)=\xi\} .
$$

Proposition 3.2 concerns only the $a_{i}^{*}$ value at a steady state. In general, several $P_{i j}^{*}$ values can be compatible with the homogeneous auxin $a_{i}^{*}=a^{*}$. Among these, the uniform zero value is always possible.

Proposition 3.3. (i) Equations (2.3)-(2.4) always admit the homogeneous steady-state solution $E_{0}=\left(a^{*} \ldots a^{*}, 0 \ldots 0\right)$ with

$$
a_{i}^{*}=a^{*}=\frac{\kappa}{\delta}, \quad P_{i j}^{*}=0, \quad i \in \mathcal{V}, j \in \mathcal{N}_{i} .
$$

Copyright $\odot$ by SIAM. Unauthorized reproduction of this article is prohibited. 
(ii) Furthermore, $E_{0}$ is the only steady state such that all $P_{i j}^{*}$ 's have the same value.

Proof. When $a_{i}^{*}=a^{*}$ and $P_{i j}^{*}=0$ for all $i \in \mathcal{V}, j \in \mathcal{N}_{i}, E_{0}$ is easily seen to be a steady state. Therefore, (i) holds.

To prove (ii), suppose now that there is a steady state such that for all $i, j$, one has $P_{i j}^{*}=P^{*}$ for a constant $P^{*} \geq 0$. From Proposition 3.1, either $P_{i j}^{*}=0$ or $P_{j i}^{*}=0$ for any steady-state solution. Thus, one must have $P^{*}=0$, which implies $\Phi_{i j}^{*}=D\left(a_{i}^{*}-a_{j}^{*}\right)$.

Let the matrix $L=\left(L_{i j}\right)$ denote the (generalized) Laplacian of the graph $\mathcal{G}$, defined more explicitly by

$$
L_{i j}=\left\{\begin{array}{cl}
\frac{-A_{i j} D}{V_{i}} & \text { if } j \neq i, \\
D \sum_{k=1}^{N} \frac{A_{i k}}{V_{i}} & \text { if } j=i
\end{array}\right.
$$

and let $\hat{a}=\left(a_{1}^{*}, \ldots, a_{N}^{*}\right)^{T}$ be the vector of auxin concentrations. Then all the auxin $a_{i}^{*}$ in the first equation of (3.1) can be read as

$$
\left(L+\delta I_{N}\right) \hat{a}=\kappa u,
$$

where $I_{N}$ is the $N \times N$ identity matrix and $u=(1, \ldots, 1)^{T} \in \mathbb{R}^{N}$. Since all rows in the matrix $L$ sum to zero, i.e., $L u=0$, and its nondiagonal elements are nonpositive, $L$ is a singular $M$-matrix (see Chapter 6 of [3] for a detailed account). Then, for any positive $\delta$, the matrix $L+\delta I_{N}$ is strictly diagonally dominant, and thus it is nonsingular. Hence, (3.7) admits a unique solution $\hat{a}=\kappa\left(L+\delta I_{N}\right)^{-1} u$. Thus, $\left(L+\delta I_{N}\right) u=\delta u$, i.e., $\left(L+\delta I_{N}\right)^{-1} u=\frac{1}{\delta} u$, so that the unique solution is given more simply by $\hat{a}=\frac{\kappa}{\delta} u$, i.e., $a_{i}^{*}=a^{*}=\frac{\kappa}{\delta}(i \in \mathcal{V})$.

Proposition 3.3 indicates that homogeneous transporter patterns necessarily induce homogeneous auxin patterns. However, the converse is not always true. Actually, if 0 is the only fixed point of $\widehat{h}$, i.e., $\mathcal{P}^{*}=\{0\}$, then homogeneous auxin at a steady state can occur only for the homogeneous equilibrium $E_{0}$. But if $\mathcal{P}^{*}$ contains at least one positive element, then the homogeneous distribution of auxin at an equilibrium is possible with nonzero (and nonhomogeneous) transporter distribution. Therefore, besides $E_{0}$, there exist nonzero steady states $P_{i j}^{*}$ that are compatible with a homogeneous auxin value $a^{*}$. Although an explicit formula for these $P_{i j}^{*}$ is difficult to obtain in general, from Proposition 3.1 we know that, for all $i \in\{1, \ldots, N\}$ and $j \in \mathcal{N}_{i}$, either (i) $P_{i j}^{*} \in \mathcal{P}^{*}$ and $P_{j i}^{*}=0$, or (ii) $P_{j i}^{*} \in \mathcal{P}^{*}$ and $P_{i j}^{*}=0$. Furthermore, we have the following proposition.

Proposition 3.4. A vector $\left(a^{*}, \ldots, a^{*},\left(P_{i j}^{*}\right)_{i, j}\right)$ is a steady state with homogeneous auxin $a^{*}$ if and only if the following holds:

(C) For all $i \in\{1, \ldots, N\}$,

$$
\sum_{k=1}^{m} \sum_{j \in J_{k}^{+}(i)} A_{i j} \mathcal{P}_{k}^{*}=\sum_{k=1}^{m} \sum_{j \in J_{k}^{-}(i)} A_{i j} \mathcal{P}_{k}^{*}
$$

holds, where $J_{k}^{+}(i)=\left\{j \in\{1, \ldots, N\}: P_{j i}^{*}=\mathcal{P}_{k}^{*}\right\}, J_{k}^{-}(i)=\left\{j \in\{1, \ldots, N\}: P_{i j}^{*}=\mathcal{P}_{k}^{*}\right\}$, and $k \in\{1, \ldots, m\}$.

Proof. With $a_{i}^{*}=a^{*}$, the first equation in (3.1) becomes

$$
\frac{1}{\delta V_{i}} \sum_{j=1}^{N} A_{i j} \Phi_{j i}^{*}=0 .
$$

Copyright $\odot$ by SIAM. Unauthorized reproduction of this article is prohibited. 
From $\Phi_{j i}^{*}=\left(P_{j i}^{*}-P_{i j}^{*}\right) a^{*}, \Phi_{j i}^{*}$ is of the form $\pm a^{*} \mathcal{P}_{k}^{*}$ for some $k \in\{0, \ldots, m\}$. Since $\frac{a^{*}}{\delta V_{i}} \neq 0$, the result is obvious.

Proposition 3.4 characterizes $P_{i j}^{*}$ values by the fact that they must belong to the finite set $\mathcal{P}^{*}$ and satisfy a constraint that depends on the matrix $A_{i j}$. This constraint can be interpreted geometrically as follows: for each $i$, the vector $v_{i}=\left(\operatorname{sign}\left(\phi_{j i}^{*}\right) P_{j i}^{*}\right)_{j}$ must be orthogonal to the row vector $\left(A_{i j}\right)_{j}$. Since $v_{i}$ can take a finite number of values, with coordinates in $\pm \mathcal{P}^{*}$ when $j \in \mathcal{N}_{i}$, and zero for other $j$, the valid $P_{i j}^{*}$ 's are defined by intersections of finite sets of vectors in $\mathbb{R}^{N}$ with hyperplanes orthogonal to the row vectors of the matrix $A$ (or its columns since it is symmetric).

Next, we can investigate the stability of $E_{0}$ for a large class of functions $h$, including the cases $n>1$ for the functions $h_{1}$ and $h_{2}$ given earlier, and in fact any function $h$ of class $C^{1}$ that satisfies our hypotheses.

Proposition 3.5. Suppose that the function $h$ satisfies

$$
h^{\prime}(0)=0 .
$$

Then, the steady state $E_{0}$ is locally asymptotically stable.

Proof. Denote $J_{0}$ the $(N+d) \times(N+d)$ Jacobian matrix of the system $(2.3)-(2.4)$, evaluated at $E_{0}$. Then the characteristic equation is $\Delta_{J_{0}}(\lambda)=\operatorname{det}\left(\lambda I-J_{0}\right)$ (where $I$ is the identity matrix of the same size). From the proof of Proposition 3.3, we know that $\Phi_{i j}^{*}=0$ for all $i, j$ at $E_{0}$. With the condition $h^{\prime}(0)=0$, we can write $J_{0}$ in block form as

$$
J_{0}=\left[\begin{array}{c|c}
-\left(L+\delta I_{N}\right) & M \\
\hline 0 & -\mu I_{d}
\end{array}\right],
$$

where $M$ is the rectangular $N \times d$ matrix corresponding to the partial derivatives with respect to $P_{i j}$ for (2.3). Assume now that the $P_{i j}$ variables are ordered. Then the terms in the $i$ th row of $M$ that correspond to the columns of $P_{i j}$ and $P_{j i}$ are respectively equal to $-\frac{A_{i j}}{V_{i}} a^{*}$ and $\frac{A_{i j}}{V_{i}} a^{*}$. For instance, in a ring of three cells, with the $P_{i j}$ ordered as $P_{12}, P_{23}, P_{31}, P_{13}, P_{32}, P_{21}$, the matrix $M$ is

$$
\left[\begin{array}{rrrrrr}
-\frac{A_{12}}{V_{1}} a^{*} & 0 & \frac{A_{13}}{V_{1}} a^{*} & -\frac{A_{13}}{V_{1}} a^{*} & 0 & \frac{A_{12}}{V_{1}} a^{*} \\
\frac{A_{12}}{V_{2}} a^{*} & -\frac{A_{23}}{V_{2}} a^{*} & 0 & 0 & \frac{A_{23}}{V_{2}} a^{*} & -\frac{A_{12}}{V_{2}} a^{*} \\
0 & \frac{A_{23}}{V_{3}} a^{*} & -\frac{A_{13}}{V_{3}} a^{*} & \frac{A_{13}}{V_{3}} a^{*} & -\frac{A_{23}}{V_{3}} a^{*} & 0
\end{array}\right] .
$$

In fact, $\Delta_{J_{0}}(\lambda)$ is independent of $M$ and reads

$$
\Delta_{J_{0}}(\lambda)=\Delta_{-\left(L+\delta I_{N}\right)}(\lambda) \Delta_{-\mu I_{d}}(\lambda)=\Delta_{-\left(L+\delta I_{N}\right)}(\lambda)(\lambda+\mu)^{d} .
$$

From the proof in Proposition 3.3, we know that, for positive $\delta, L+\delta I_{N}$ is a nonsingular $M$ matrix, so all of its eigenvalues have positive real part. Consequently, all roots of $\Delta_{-\left(L+\delta I_{N}\right)}$ must have negative real part. Together with the negative eigenvalue $-\mu$ of order $d$, the proof is complete.

Remark 1. One important consequence of Proposition 3.5 is that no auxin pattern can emerge from a destabilization due to small perturbations of the steady state $E_{0}$ when $h^{\prime}(0)=0$. 
It is possible to prove that this property also holds for the model (2.3), (2.5)-(2.6) including PIN reallocation. From the discussion after (2.5)-(2.6) it follows that this alternative model has a steady state where all $P_{i j}=0$ and all $a_{i}=a^{*}$ like $E_{0}$, and $P_{i}=\rho_{P} / \delta_{P}$ for all $i$. Let us denote this steady state by $\widetilde{E}_{0}$. Then, as in the proof above, a direct calculation shows that if $h^{\prime}(0)=0$, the Jacobian matrix of the model (2.3), (2.5)-(2.6) at $\widetilde{E}_{0}$ has the form

$$
\left[\begin{array}{c|c|c}
-\left(L+\delta I_{N}\right) & 0 & M \\
\hline 0 & -\varepsilon \delta_{P} I_{N} & Q \\
\hline 0 & 0 & -\mu I_{d}
\end{array}\right],
$$

where $M$ is exactly as in the proof of Proposition 3.5 and $Q$ represents derivatives of the right-hand side of (2.5) with respect to $P_{i j}$ variables. Though $Q$ can be made more explicit, the block-triangular form of the Jacobian allows us to conclude directly that $\widetilde{E}_{0}$ is locally asymptotically stable, like $E_{0}$.

As discussed earlier, for the functions $h_{1}$ and $h_{2}$ with $n=1$, the condition $h^{\prime}(0)=0$ does not hold. In such a case, a standard stability analysis method using the eigenvalues of the Jacobian matrix cannot be performed in a straightforward way. Indeed, the Jacobian is discontinuous on the union of hypersurfaces of the form

$$
S_{i j}=\left\{(a, P) \in \mathbb{R}^{N+d}: \Phi_{i j}=\left(P_{i j}+D\right) a_{i}-\left(P_{j i}+D\right) a_{j}=0\right\} .
$$

In particular, the equilibrium $E_{0}$ is situated at the intersection of all these surfaces, since it satisfies $\Phi_{i j}^{*}=0$ for all $i, j$. In this case the analysis is more involved and will be considered only for particular examples in the following section.

For a steady state such that $\Phi_{i j}^{*} \neq 0$, on the other hand, a standard stability analysis is still possible. This follows from the fact that the half spaces defined by

$$
S_{i j}^{ \pm}=\left\{(a, P) \in \mathbb{R}^{N+d}: \pm \Phi_{i j}= \pm\left(\left(P_{i j}+D\right) a_{i}-\left(P_{j i}+D\right) a_{j}\right)>0\right\}
$$

are open subsets of $\mathbb{R}^{N+d}$. Hence a steady state that lies inside the subspaces of this form will always have an open neighborhood in which the Jacobian varies continuously.

3.2. Regular tissues. In this section we will consider only idealized tissues, in order to get a better understanding of the patterns expected to occur in the system (2.2)-(2.4). Throughout the section we assume that all cells have the same volume $V_{i}=V$ and identical contact surface area $A_{i j}=A$. Then the equations can be simplified further, thanks to the following rescaling:

$$
\widetilde{P_{i j}}=\frac{A}{V} P_{i j}, \quad \widetilde{D}=\frac{A}{V} D, \quad \widetilde{h}(\Phi)=\frac{A}{V} h\left(\frac{V}{A} \Phi\right) .
$$

Omitting the tildes, (2.3)-(2.4) become

$$
\begin{aligned}
\frac{d a_{i}}{d t} & =\sum_{j \in \mathcal{N}_{i}} \Phi_{j i}+\kappa-\delta a_{i}, \\
\frac{d P_{i j}}{d t} & =h\left(\Phi_{i j}\right)-\mu P_{i j},
\end{aligned}
$$

Copyright $\odot$ by SIAM. Unauthorized reproduction of this article is prohibited. 
where $\Phi_{i j}$ is defined as in (2.2).

As discussed in the previous subsection, with homogeneous auxin the value of $P_{i j}^{*}$ in a steady solution is determined by the nonnegative fixed points of the function $\widehat{h}$ in (3.5) which always contains $P_{0}^{*}=0$. If 0 is the only value in $\mathcal{P}^{*}$, then $E_{0}$ is clearly the only steady state with homogeneous auxin distribution, implying that the only asymptotic PIN pattern would be zero PIN everywhere on the tissue. This is not very relevant from a biological point of view.

Suppose now that $\widehat{h}$ admits a unique positive fixed point; then Proposition 3.1 allows us to determine the values $P_{i j}^{*}$ when all $a_{i}^{*}$ are fixed to $a^{*}$ at a steady state.

Proposition 3.6. Consider the system (3.9), (3.10). Suppose that there exists a unique positive fixed point $\mathcal{P}_{1}^{*}$ to the function $\widehat{h}$, i.e., $\mathcal{P}^{*}=\left\{0, \mathcal{P}_{1}^{*}\right\}$. Then, there exists a steady state with homogeneous auxin concentration, i.e., $a_{i}^{*}=a^{*}$ for all $i \in\{1, \ldots, N\}$, if and only if both of the following conditions are true:

(a) for all $i, j,\left(P_{i j}^{*}, P_{j i}^{*}\right) \in\left\{(0,0),\left(0, \mathcal{P}_{1}^{*}\right),\left(\mathcal{P}_{1}^{*}, 0\right)\right\}$;

(b) for all $i, \operatorname{card}\left(\left\{j \in \mathcal{N}_{i}: P_{i j}^{*}=\mathcal{P}_{1}^{*}\right\}\right)=\operatorname{card}\left(\left\{j \in \mathcal{N}_{i}: P_{j i}^{*}=\mathcal{P}_{1}^{*}\right\}\right)$.

Proof. From Proposition 3.2, one deduces that there exists a steady state with homogeneous auxin if and only if $a_{i}^{*}=a^{*}$ for all $i$, and the $P_{i j}^{*}$ 's satisfy condition (a), from Propositions 3.1 and 3.3. Moreover, the $P_{i j}^{*}$ 's must be zeros of (3.10) such that $\sum_{j \in \mathcal{N}_{i}} \Phi_{j i}^{*}=0$ from (3.9), which can be rewritten as

$$
\sum_{\substack{j \in \mathcal{N}_{i} \\ \Phi_{j i}^{*}>0}} \Phi_{j i}^{*}=-\sum_{\substack{j \in \mathcal{N}_{i} \\ \Phi_{j i}^{*}<0}} \Phi_{j i}^{*} .
$$

Since $\Phi_{i j}^{*}=a^{*}\left(P_{i j}^{*}-P_{j i}^{*}\right)$, from Proposition 3.1 and the assumption on $\widehat{h}$, it follows that $\left(P_{i j}^{*}, P_{j i}^{*}\right) \in\left\{(0,0),\left(0, \mathcal{P}_{1}^{*}\right),\left(\mathcal{P}_{1}^{*}, 0\right)\right\}$, and thus $\Phi_{i j}^{*} \in\left\{0,-a^{*} \mathcal{P}_{1}^{*}, a^{*} \mathcal{P}_{1}^{*}\right\}$, with $\Phi_{i j}^{*}>0$ if and only if $P_{i j}^{*}=\mathcal{P}_{1}^{*}$. Equality (3.11) is equivalent to condition (b) in Proposition 3.6.

One may remark here that the conditions provided in Proposition 3.6 can be stated quite simply in terms of the directed graph $\mathcal{G}^{+}\left(\Phi^{*}\right)$ at a steady state with $i \rightarrow j$ if and only if $\Phi_{i j}^{*} \geq 0$. More specifically, an edge $i-j$ between $i, j$ is directed in $\mathcal{G}^{+}\left(\Phi^{*}\right)$ as

- $i \rightarrow j$ if and only if $P_{i j}^{*}=\mathcal{P}_{1}^{*}$, and thus $P_{j i}^{*}=0$,

- $j \rightarrow i$ if and only if $P_{j i}^{*}=\mathcal{P}_{1}^{*}$, and thus $P_{i j}^{*}=0$, and

- $i \leftrightarrow j$ if and only if $P_{i j}^{*}=P_{j i}^{*}=0$.

Then, condition (b) of Proposition 3.6 is equivalent to the fact that every node in $\mathcal{G}^{+}\left(\Phi^{*}\right)$ has the same number of incoming and outgoing edges. This type of directed graph is often called balanced in the literature (see, e.g., [1]). To take advantage of this formulation in what follows, let us define $\mathcal{N}_{i}^{+}$(resp., $\mathcal{N}_{i}^{-}$) as the set of incoming (resp., outgoing) edges at node $i$ in $\mathcal{G}^{+}\left(\Phi^{*}\right){ }^{1}$

In particular, one can consider a simple ring of cells. In this case, indeed, the conditions in Proposition 3.6 are immediately verified.

\footnotetext{
${ }^{1}$ It is often the case in the graph theory literature that in and out neighbors are denoted using a $-/+$ sign, respectively. Our choice to use the opposite notation is motivated by the sign of auxin flux on edges, which will be positive for incoming edges.
}

Copyright @ ( by SIAM. Unauthorized reproduction of this article is prohibited. 
Example 1. Assume a tissue has the structure of a ring of $N$ cells indexed from 1 to $N$ clockwise, with the nearest neighboring connection. Then $\mathcal{N}_{i}=\{i-1, i+1\}$ for all $i \bmod (N)$. Denote the index of $P_{i j}$ in the following order:

$$
\left(P_{12}, P_{23}, \ldots, P_{i, i+1}, \ldots, P_{N 1}, P_{1 N}, P_{N, N-1}, \ldots, P_{i, i-1}, \ldots, P_{21}\right) \text {. }
$$

Then an orientation of the graph $\mathcal{G}$ can be balanced in exactly three ways:

(a) Undirected ring: for all $i \in\{1, \ldots, N\}, \mathcal{N}_{i}^{-}=\mathcal{N}_{i}^{+}=\varnothing$.

Corresponding steady state: $E_{0}=\left(a^{*}, \ldots, a^{*}, 0, \ldots, 0,0, \ldots, 0\right)$.

(b) Anticlockwise flux: for all $i \in\{1, \ldots, N\}, \mathcal{N}_{i}^{-}=\{i+1\}$ and $\mathcal{N}_{i}^{+}=\{i-1\}$.

Corresponding steady state: $E_{+}=\left(a^{*}, \ldots, a^{*}, \mathcal{P}_{1}^{*}, \ldots, \mathcal{P}_{1}^{*}, 0, \ldots, 0\right)$.

(c) Clockwise flux: for all $i \in\{1, \ldots, N\}, \mathcal{N}_{i}^{-}=\{i-1\}$ and $\mathcal{N}_{i}^{+}=\{i+1\}$.

Corresponding steady state: $E_{-}=\left(a^{*}, \ldots, a^{*}, 0, \ldots, 0, \mathcal{P}_{1}^{*}, \ldots, \mathcal{P}_{1}^{*}\right)$.

This associates, respectively, to an absence of flux for the first case, or a constant flux circulating from each cell to the neighbor cell with greater (resp., lower) index in $\{1, \ldots, N\}$ for the second (resp., third) case; see Figure 3 for an illustration.

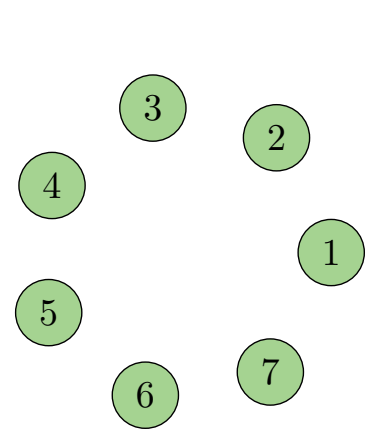

(a)

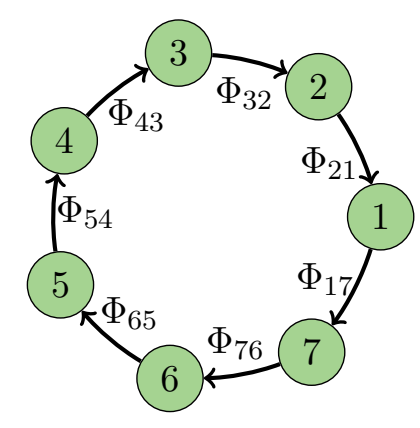

(b)

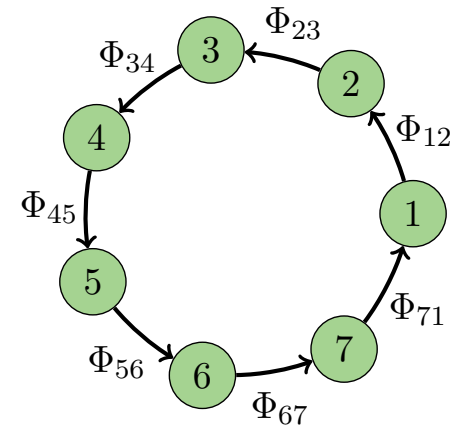

(c)

Figure 3. A ring of $N$ cells, with the three possible balanced orientations. $N=7$.

The steady state $E_{0}$ defined in Proposition 3.3 together with the steady states characterized in Proposition 3.6 describe all the steady states with homogeneous auxin under the assumption that $\widehat{h}$ admits at most one positive equilibrium. This assumption holds for a number of functions, including $h_{1}$ for $n \leqslant 1$ or $h_{2}$ for any exponent $n$. The function $h_{2}$ with $n>1$ may typically have two positive equilibria, which suggests that an extension of Proposition 3.6 would be useful, while the patterns would be more complicated; this is outside the scope of this article.

We have seen in Proposition 3.5 that the equilibrium patterns with uniformly zero $P_{i j}^{*}$ are locally asymptotically stable whenever the flux dependent rate $h$ has zero derivative at the origin. Hence, it remains for us to consider (i) the stability of $E_{0}$ when $h^{\prime}\left(0^{+}\right)>0$ and (ii) the steady states where some $P_{i j}^{*}$ are nonzero for $h^{\prime}\left(0^{+}\right) \geq 0$.

In case (i), since at $E_{0}$ all the fluxes $\Phi_{i j}^{*}=0$, the Jacobian is not continuous in a neighborhood of $E_{0}$, and a local stability analysis cannot rely on this matrix, as discussed at the 
end of section 3.1. A similar difficulty will remain true in case (ii) for $h^{\prime}\left(0^{+}\right)>0$ as long as at least one pair $i, j$ is such that $\Phi_{i j}^{*}=0$. In the particular case of a ring of cells, however, for the steady states $E_{-}$and $E_{+}$, all $\Phi_{i j}^{*}$ equal either $-a^{*} \mathcal{P}_{1}$ or $a^{*} \mathcal{P}_{1}$ and thus are nonzero. It follows that the Jacobian is continuous at least in a neighborhood of $E_{-}$and $E_{+}$and that their stability can be deduced from the eigenvalues of this Jacobian.

To simplify the analysis, we consider the stability of homogeneous equilibria in the case of a ring of three cells, that is, the system (3.9)-(3.10) with the structure given in Example 1. Since there exist three equilibrium solutions $E_{0}, E_{+}$, and $E_{-}$, we denote the corresponding Jacobian matrix by $J_{0}, J_{+}$, and $J_{-}$, respectively. The detailed computation of these matrices is given in Appendix A.

At the steady state $E_{0}$, we know from Appendix A that, when $h^{\prime}(0)=0$, the characteristic equation is

$$
\Delta_{J_{0}}(\lambda)=(\lambda+\delta)(\lambda+\mu)^{4}\left(\lambda^{2}+(3 D+\delta+\mu) \lambda+\mu(3 D+\delta)\right)^{2} .
$$

It is easy to see that all the eigenvalues in $J_{0}$ are negative. Therefore, $E_{0}$ is locally asymptotically stable, which is consistent with the prediction given by Proposition 3.5.

When $h^{\prime}\left(0^{+}\right)>0$, i.e., $h^{\prime}(0)$ does not exist, any neighborhood near $E_{0}$ contains discontinuity surfaces for $h^{\prime}$, of the form $S_{i j}$ given in (3.8); hence trajectories in such a neighborhood may in general cross one of these surfaces, changing the dynamical behavior. As a consequence, the following computations at $E_{0}$ cannot provide us with rigorous results on the stability. However, we will use them as a heuristic for numerical simulations performed in next section. With $h^{\prime}\left(0^{+}\right)>0$, the characteristic equation becomes

$$
\Delta_{J_{0}}(\lambda)=(\lambda+\delta)\left(\lambda-\frac{h^{\prime}\left(0^{+}\right) \kappa-\delta \mu}{\delta}\right)^{4}\left(\lambda^{2}-H \lambda-\kappa h^{\prime}\left(0^{+}\right)+\mu(3 D+\delta)\right)^{2},
$$

where

$$
H=\frac{h^{\prime}\left(0^{+}\right) \kappa}{\delta}-(3 D+\delta+\mu)
$$

When $h^{\prime}\left(0^{+}\right) \kappa<\delta \mu, H<\mu-(3 D+\delta+\mu)=-(3 D+\delta)<0$, and the constant term in the quadratic factor is $-\kappa h^{\prime}\left(0^{+}\right)+\mu(3 D+\delta)>-\delta \mu+\mu(3 D+\delta)=3 D \mu>0$, suggesting that $E_{0}$ could still be locally asymptotically stable and become unstable for the reversed inequality. At $h^{\prime}(0) \kappa=\delta \mu$, all the eigenvalues of $J_{0}$ are negative except $\lambda=0$, indicating a fold bifurcation at $E_{0}$. Further, in an extreme case, when $\kappa h^{\prime}(0)-\mu(3 D+\delta)<0$ and $H=0$, for some particular parameter values, Hopf bifurcation may take place. More specifically, when $\mu>\delta+\frac{\delta^{2}}{3 D}$, the system undergoes a Hopf bifurcation, implying the appearance of oscillation behavior.

Let us now briefly discuss the stability of equilibria $E_{-}$and $E_{+}$analytically. These two steady states are clearly equivalent, up to a permutation of the variables, and, in particular, they have the same characteristic equation,

$$
\Delta_{J_{ \pm}}(\lambda)=(\lambda+\delta)(\lambda+\mu)^{3}\left(\lambda+\mu-a^{*} h_{*}^{\prime}\right) \widetilde{\Delta}(\lambda)
$$

where

$$
\widetilde{\Delta}(\lambda)=\lambda^{4}+c_{3} \lambda^{3}+c_{2} \lambda^{2}+c_{1} \lambda+c_{0}
$$

Copyright ( ) by SIAM. Unauthorized reproduction of this article is prohibited. 
with

$$
\begin{aligned}
c_{3}= & 2\left(\mu-a^{*} h_{*}^{\prime}\right)+6 D+2 \delta+3 P_{1}^{*}, \\
c_{2}= & \left(\mu-a^{*} h_{*}^{\prime}\right)\left[3 P_{1}^{*}+6 D+4 \delta+\left(\mu-a^{*} h_{*}^{\prime}\right)\right]+6 D \mu+3 \mu P_{1}^{*} \\
& +3 P_{1}^{* 2}+9 D^{2}+\delta^{2}+9 D P_{1}^{*}+3 \delta P_{1}^{*}+6 D \delta, \\
c_{1}= & \left(\mu-a^{*} h_{*}^{\prime}\right)\left[2 \delta^{2}+3 \mu P_{1}^{*}+2 \delta\left(\mu-a^{*} h_{*}^{\prime}\right)+6 D \mu+6 D \delta+3 \delta P_{1}^{*}\right] \\
& +3 \mu\left(2 D \delta+6 D P_{1}^{*}+\delta P_{1}^{*}+6 D^{2}+2 P_{1}^{* 2}\right), \\
c_{0}= & \left(\mu-a^{*} h_{*}^{\prime}\right)\left[6 D \mu \delta+3 \mu \delta P_{1}^{*}+\delta^{2}\left(\mu-a^{*} h_{*}^{\prime}\right)\right]+3 \mu^{2}\left(3 D P_{1}^{*}+3 D^{2}+P_{1}^{* 2}\right) .
\end{aligned}
$$

When $a^{*} h_{*}^{\prime}<\mu$, that is, $\kappa h_{*}^{\prime}<\mu \delta$, all the coefficients $c_{i}>0$ for all $i=0, \ldots, 3$. In addition, if $c_{2} c_{3}-c_{1}>0$ and $c_{1} c_{2} c_{3}-c_{1}^{2}-c_{0} c_{3}^{2}>0$, we can conclude that the steady states $E_{ \pm}$are locally asymptotically stable from Hurwitz criteria. Actually, we can compute

$$
c_{2} c_{3}-c_{1}=\left(\mu-a^{*} h_{*}^{\prime}\right)\left[2\left(\mu-a^{*} h_{*}^{\prime}\right)^{2}+\left(\mu-a^{*} h_{*}^{\prime}\right)\left(8 \delta+9 P_{1}^{*}+18 D\right)+P \cdot T_{1}\right]+P \cdot T_{2},
$$

where $P . T_{i}(i=1,2)$ stands for the positive terms, while we omit the tedious calculation of $c_{1} c_{2} c_{3}-c_{1}^{2}-c_{0} c_{3}^{2}$.

Similarly, Hopf bifurcation is also possible, for instance, when $c_{0}=0, c_{1}=c_{2} c_{3}$, and $c_{2}>0$. Due to the complexity, we cannot provide explicit conditions, while the analysis on each steady state strongly suggests that the systems may present Hopf bifurcation. When the Hopf bifurcation is supercritical, we should be able to observe the stable periodic solutions numerically.

4. Numerical results. In this section we present numerical simulations to confirm the theoretical results obtained previously and to explore rich dynamical behavior beyond the analytical domain.

First, we consider a tissue with a ring of $N$ cells and choose the function $h(x)=\frac{x^{5}}{5^{5}+x^{5}} \chi_{[x>0]}$. Theoretically, we know the equilibrium $E_{0}$ is stable from Proposition 3.5 since $h^{\prime}(0)=0$. With the parameters $(D, \delta, \kappa, \mu)=(0.1,1,1,1)$, when $N=3, \widehat{h}$ has two positive fixed points, and hence Proposition 3.6 cannot be applied directly, while the steady states of both $E_{+}$and $E_{-}$ are observed numerically in Figure 4 by using various, randomly selected, initial conditions. This illustrates the fact that the analytical results obtained in the previous section are likely to be valid under weaker assumptions.

Although we previously discussed only the steady states with homogeneous auxin, auxin is not always distributed uniformly in real tissues, especially for a tissue with a large number of cells. Figure 5 shows some of the steady states with a different number of cells in a ring.

From Figure 5, we can see that the steady states typically involve local maxima of auxin, surrounded by a sharp decrease of auxin in the neighboring cells (for the tested parameters). Biologically, such patterns are reminiscent of the local auxin maxima observed in meristems at the locations where new organs are emerging. In particular, the formation of flowers is known to imply ring-shaped regions where specific organs (sepals, petals, stamens, and carpel) are formed. Another interpretation of these patterns is that they correspond to the spontaneous emergence of sources and sinks of auxin: sinks are cells with high auxin and incoming flux, whereas sources are groups of cells with low auxin and outgoing flux. A general trend in our

Copyright $\odot$ by SIAM. Unauthorized reproduction of this article is prohibited. 


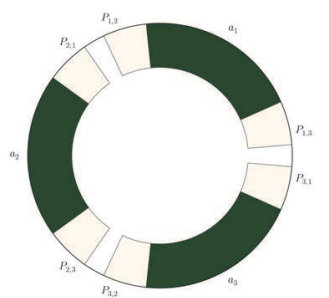

(a)

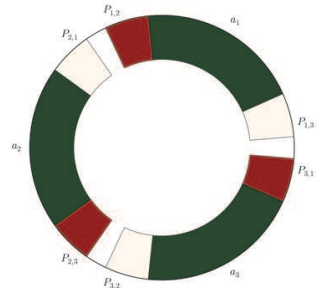

(b)

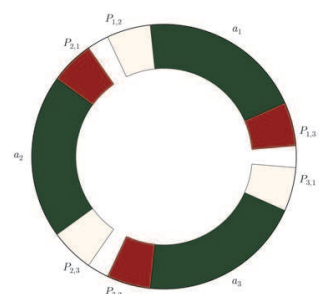

(c)

Figure 4. Representation of three steady-state solutions with different (randomly generated) initial conditions, in a ring of three cells. (a) Stable equilibrium $E_{0}$; (b) stable $E_{+}$; (c) stable $E_{-}$.

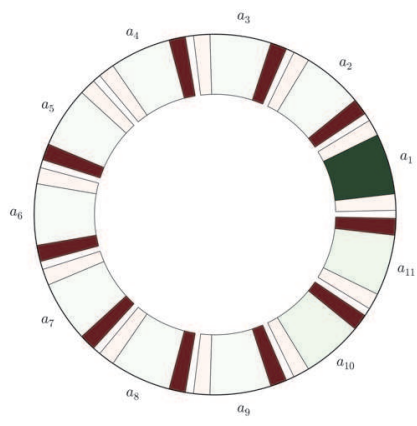

(a)

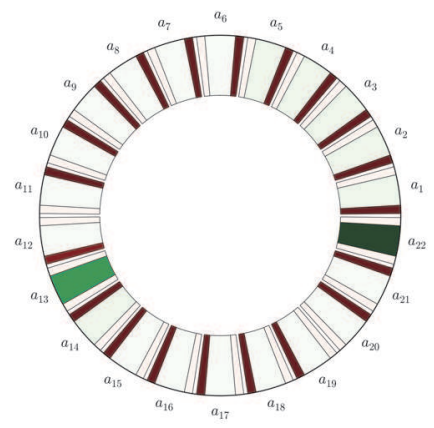

(b)

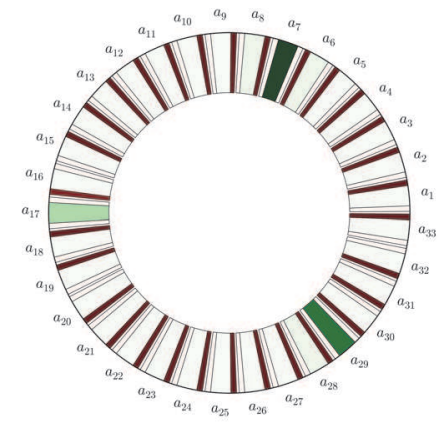

(c)

Figure 5. Steady-state solutions with a different number of cells in a ring; the parameters $(D, \delta, \kappa, \mu)=$ (0.1,1,10,0.1). (a) $N=11$, (b) $N=22$, and (c) $N=33$. Because small differences in concentration are difficult to visualize using a mere colormap, we mention that cells (resp., sides) with light green (resp., light red) colors represent essentially uniform auxin (resp., PIN) concentrations, close to zero.

simulations was that sources typically involved groups of at least two cells, whereas sinks were more often localized at single cells. Similar simulations using a reallocation model of the form (2.5)-(2.6) led to essentially the same patterns.

Due to the complexity of expressions shown at the end of the previous section, our exploration of Hopf bifurcations near the steady states $E_{ \pm}$was not conclusive. However, we numerically discovered the possibility of oscillations through a destabilization of $E_{0}$ when $h$ is not smooth enough (say, $h \notin C^{1}$ ). We started with a simple case of two neighboring cells and chose $h(x)=\rho x$ for $x>0$ (and 0 otherwise). Similar to the three-cell ring, mathematically we know that, when $h^{\prime}\left(0^{+}\right) \kappa<\delta \mu, E_{0}$ should be locally asymptotically stable and become unstable when $h^{\prime}\left(0^{+}\right) \kappa>\delta \mu$; if $h^{\prime}\left(0^{+}\right) \kappa=\delta(2 D+\delta+\mu)$ and $\mu>\delta+\frac{\delta^{2}}{2 D}$, oscillation is possible via Hopf bifurcation. Actually, for the parameters $(D, \delta, \kappa, \mu)=(0.3,0.5,0.5,1$. , when $h^{\prime}\left(0^{+}\right)=\rho<\frac{\mu \delta}{\kappa}=0.3$, through the numerical simulation, the stability of $E_{0}$ is easy to see. However, we can observe that the stability of $E_{0}$ remains with $\rho=2.0$ (Figure 6). This confirms that the analytical result is unreliable due to the lack of continuity in the Jacobian matrix. It is also interesting to observe that the increase in $\rho$ leads to alternative asymptotic behavior. For instance, oscillation takes place when $\rho>2$, the periodic solution disappears 
when $\rho>2.6$, and the steady state with nonhomogeneous auxin and transporter proteins appears. See Figure 6(b), (c).

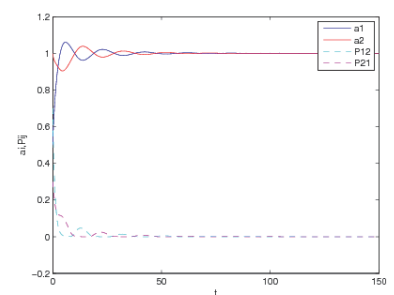

(a)

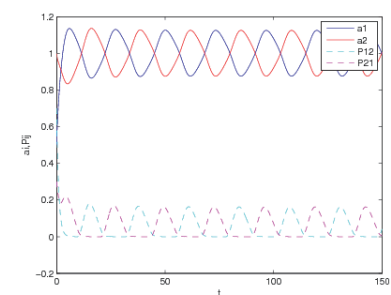

(b)

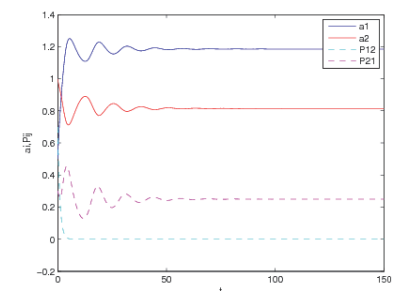

(c)

Figure 6. Solution curves in the model of two cells with different values of $\rho$ and fixed parameters $(D, \delta, \kappa, \mu)=(0.3,0.5,0.5,1$.). (a) Steady state with homogeneous auxin when $\rho=2.0$; (b) periodic solution when $\rho=2.3$; (c) steady state with nonhomogeneous auxin when $\rho=2.7$.

Although a ring with two or three cells seems analogous, unlike the case of two cells, we were not able to find any periodic solution in a ring of three cells (although this does not prove the nonexistence of such oscillation). On the other hand, we further numerically explored the dynamics in a row of more than two cells. In general, we can take as an example a tissue with a row of $N$ cells where each cell $i$ has neighbors $\mathcal{N}_{i}=\{i-1, i+1\}$ except for $\mathcal{N}_{1}=\{2\}$ and $\mathcal{N}_{N}=\{N-1\}$. When $N=10$, we can observe stable periodic solution for the parameters $(D, \delta, \kappa, \mu)=(7,0.5,0.5,1.5)$ and $h(x)=2.8 x$ for $x>0$ (and 0 otherwise). It is interesting to see that some of the trajectories in auxin are antiphased oscillations, which is reasonable due to the symmetry of the system. See Figure 7. In the row of cells, this antiphase solution takes in fact the form of a solitary wave, where a peak of auxin moves alternately from one end of the row to the other. Similar oscillations were also found for other numbers of cells $N$.

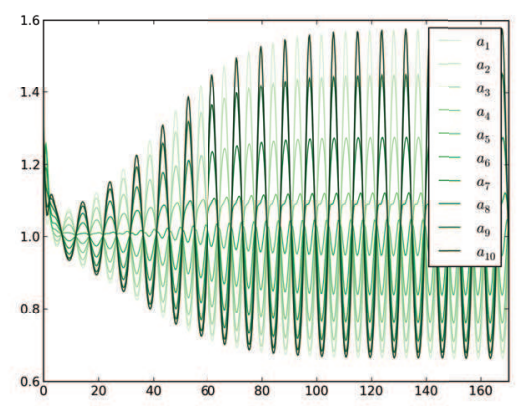

(a)

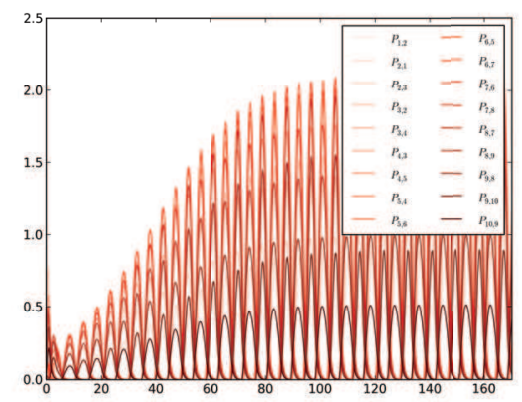

(b)

Figure 7. $N=10$, parameters $(D, \delta, \kappa, \mu)=(7,0.5,0.5,1.5)$, and $\rho=2.8$. (a) Antiphased periodic solutions in auxin. (b) Periodic solutions in transporter proteins. A video representation on a row of cells is provided (89153_01.avi [local/web 7.67MB]).

From a biological point of view, oscillations of genes responding to auxin have been observed experimentally in root tissues [22]. However, it is not entirely clear to date whether these oscillations are due to periodic evolution of auxin itself, or its biosynthesis, or even its 
signaling pathway. What the above simulations indicate is that active transport alone is susceptible to induce stable oscillations of auxin. It is also worth noticing that these oscillations have been observed in rows of cells, which are not a bad approximation of the organization of root tissues.

However, a strong difference between the row of cells studied here and a real root tissue is that the latter grows and undergoes repeated cell divisions in meristematic tissues. Since each cell division implies a change in the system's dimension, an analytical approach would amount to determining the set of parameter values for which stable oscillations occur simultaneously in rows made of increasing numbers of cells. Since the set of parameters for a fixed number of cells is already challenging to characterize, an analytic study would lead us beyond the scope of this paper. We thus used numerical simulations to investigate the robustness of our observations. We performed numerical simulations where a new cell was added at one extremity of the row of cells at regular time intervals, mimicking the fact that cell divisions in roots almost exclusively take place at the tip (with the obvious exception of lateral roots). At such events, the auxin concentration in the newly formed cell, say, cell $N+1$, was supposed to equal to that of its left-hand side neighbor (its mother cell), whereas initial values for $P_{N, N+1}$ and $P_{N+1, N}$ were set to zero.

For small rows of only two or three cells, we did not find parameters for which oscillations were maintained after the adjunction of a new cell. For larger numbers of cells, however, we observed that oscillations were typically maintained despite multiple cell divisions. As an example, Figure 8 shows a simulation where oscillations persist for a row of 10 cells which undergoes 30 successive cell division events. Since oscillations observed in $[7,22]$ had a period of $\approx 4-6 \mathrm{~h}$ and cell divisions occur with a period of $\approx 12-24 \mathrm{~h}$, we added new cells typically after two to four periods of the auxin and PIN oscillations in our simulations.

Finally, to assess the potential impact of PIN reallocation, we tested the alternative model (2.5)-(2.6) for periodic solutions. As shown in Figure 9, for 10 cells oscillations were still observed using exactly the same parameters as before. However, as we tried to implement cell division as described above, the oscillations did not persist. Attempts with different but close parameter values were not successful either. Although a more comprehensive numerical exploration of parameter values may lead to a different conclusion, this observation may be an indication that oscillatory solutions are less robust when PIN reallocation is explicitly represented in the model.

5. Conclusion and discussion. Motivated by a class of proposed models for the transport of auxin in a tissue, we consider a system where auxin is transported actively among neighboring cells as an increasing function of its local flux. In the same vein as previous studies $[8,9,23,25]$, we have used techniques of dynamics to contribute to a comprehensive analysis of steady states and patterns in the system. A specificity of flux-based models is that a given auxin distribution at steady state can be obtained for different distributions of PIN. In particular, we have proved that a trivial steady state with homogeneous auxin and no transporter protein always exists in a generic tissue, while in a regular tissue (where all cells have the same volume and contact surface to their neighbors), we have characterized the set of homogeneous auxin patterns under mild assumptions on the function $h$. More specifically, we can show that, when the flux function $h$ is smooth enough, say, $h^{\prime}(0)=0$, then the trivial

Copyright @ ( by SIAM. Unauthorized reproduction of this article is prohibited. 


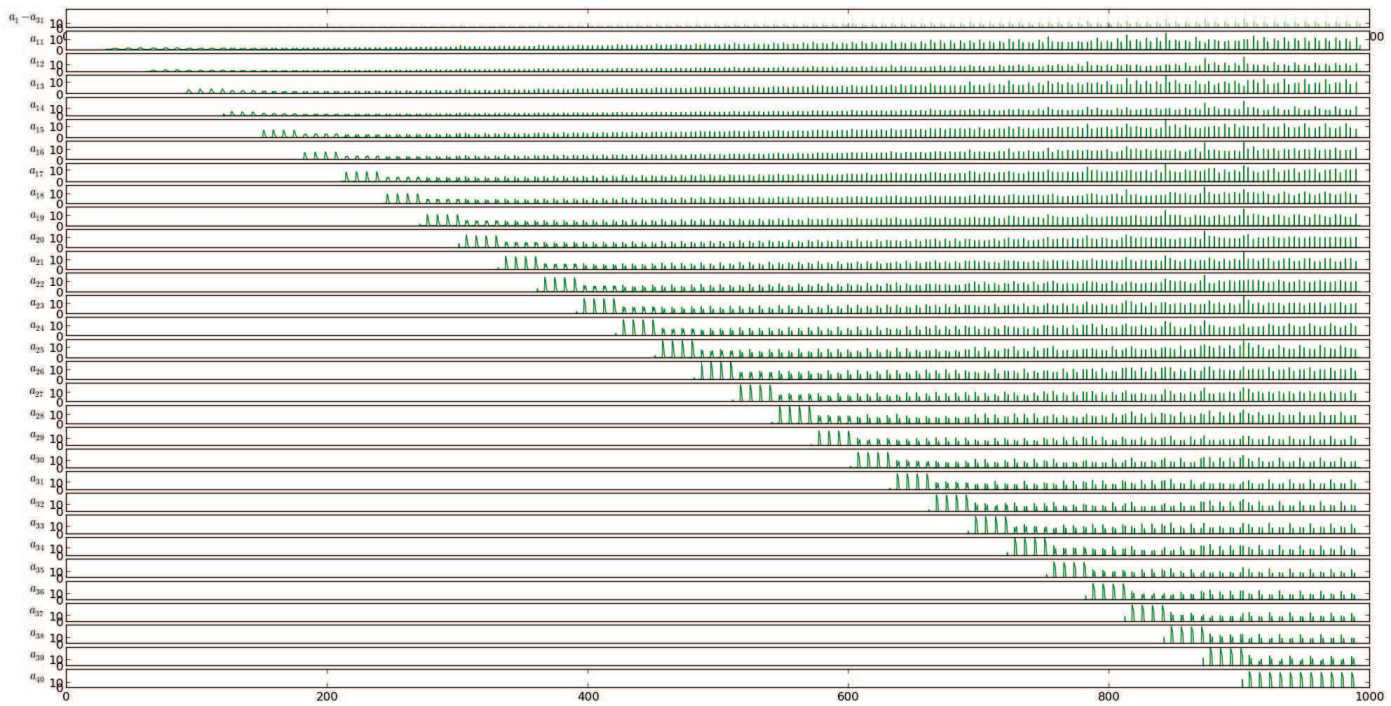

Figure 8. Row of 10 cells for which an additional cell is added periodically at the right-hand side extremity, as described in the text. Only $a_{i}$ variables are shown for sake of readability; the first row simultaneously represents all $a_{i}$ for $i \leqslant 10$. A video representation of a row of cells is provided (89153_02.avi [local/web 16.4MB]). Parameters are as in Figure 7.

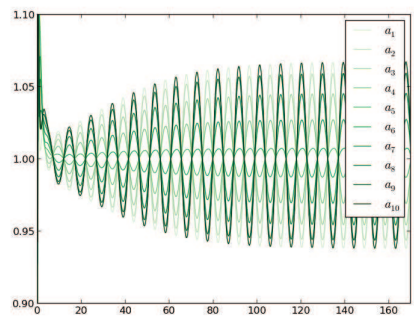

(a)

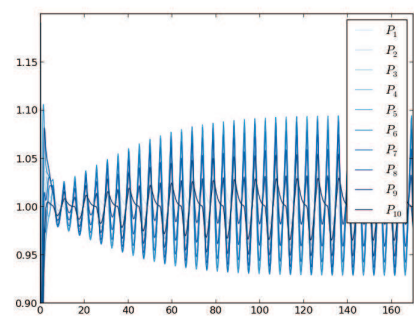

(b)

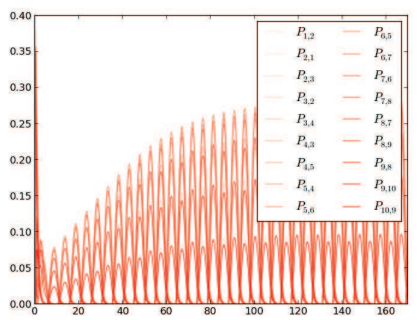

(c)

Figure 9. $\quad N=10$. Same parameters as in Figure 7, but with reallocation model (2.5)-(2.6) using $\left(\epsilon \rho_{p}, \epsilon \delta_{P}, \lambda\right)=(1,1,1)$. (a) Antiphased periodic solutions in auxin. (b)-(c) Periodic solutions in transporter proteins $P_{i}$ and $P_{i j}$, respectively.

steady state is always locally asymptotically stable, regardless of the tissue structure.

Although most results are given for flux-based models where PIN is allocated independently on the sides of each cell, we also consider a case where a limited pool of PIN proteins is available in every cell, of the form (2.5)-(2.6). Most conclusions remained valid in this context, in particular all the results concerning homogeneous steady states. The main difference was a seemingly less robust occurrence of oscillations, which did not persist in our attempts to include cell division. The similar steady states may appear to contradict the results in [10], where PIN reallocation was shown to have specific patterning properties, with high auxin in veins, whereas independent allocation led to low auxin in veins. However, there are at least two explanations for this apparent discrepancy. First, the results in [10] relied 
on numerical simulations on a fairly complex tissue, for which an exhaustive exploration of parameter regimes is impossible, and some patterns may have been missed. More likely, even though models (2.3)-(2.4) and (2.5)-(2.6) have essentially identical steady states, they may in general have different stability properties. Further work on this question may provide a mathematical explanation for the properties observed in [10].

In the particular case of a ring of cells, there are two nontrivial steady states with homogeneous auxin, corresponding respectively to a constant clockwise and counterclockwise flux. The dynamical analysis shows that these two steady states are possibly stable under certain conditions on parameters. More generally, this work shows that steady states can be characterized in graph theoretic terms, for instance by balanced orientations (see Proposition 3.6), as schematized in Figure 3 for a ring of cells. Since an increasing number of techniques are currently being developed to study complex dynamical systems using graph theoretic tools [16], a promising direction for future work would be to investigate more thoroughly the potential benefits of graph theoretic techniques to study active transport of auxin, both for concentration-based and flux-based models. It would be particularly relevant to investigate more thoroughly the nonhomogeneous steady states using this type of framework.

Through the study, we realize that the form of the flux function plays a key role in determining the dynamical behavior of the system. In particular, patterns arising through the usual Turing mechanism, where a uniform steady state loses stability [28], can never occur in the case where $h^{\prime}(0)=0$. Since patterns do occur in real tissue, our results show that this may happen in two possible ways:

- The function $h$ is not smooth.

- The function $h$ is smooth, and then patterns cannot arise from small perturbations of a uniform auxin distribution, due to the stability of this distribution. They must arise from a prepattern of auxin synthesis or decay, or from a strong perturbation.

A biological interpretation of the first possibility is that the flux activates PIN in a very "sharp" manner. Perhaps more interestingly, the second option can be interpreted as the fact that real auxin patterns do not arise from active transport alone [29]. Indeed, unless the auxin biosynthesis (or decay) is itself nonuniform, stable nonuniform steady states (i.e., patterns) are reached only after perturbations which are strong enough to lead the system's state out of the attracting basin of the uniform steady state. Even then, note that a strong perturbation is necessary but not sufficient, since it may actually lead to another steady state with uniform auxin (and nonuniform PIN). Such perturbations or prepatterns then have to be explained by additional mechanisms not represented in the model of active transport.

Different forms of $h$ will not only produce different levels of positive transporter proteins as equilibrium patterns of the system but also influence the dynamical properties of the system. For example, stable oscillations are observed numerically only for the class of $h$ functions which are not smooth at 0 . Note, however, that this does not exclude oscillations for smooth $h$ in principle. Indeed, oscillations found in this paper occur via a Hopf bifurcation at the trivial homogeneous equilibrium, and we prove that such a bifurcation can occur only for nonsmooth $h$. However, it is not possible to exclude the occurrence of a Hopf bifurcation near a nontrivial homogeneous steady state when $h$ is smooth. Verifying whether such a Hopf bifurcation can actually occur in these systems could be a topic of future work.

Oscillations in flux-based models were mentioned in the early works of Mitchison [19], 
where he termed them "parasitic oscillations" and tried to show that they would not occur in realistic parameter ranges. However, since this original work, genes activated by auxin have been experimentally observed to oscillate in roots [7, 22], though the phenomenon remains partly unclear. One possible hypothesis is that the auxin signaling pathway oscillates [17] with fixed auxin levels. One other hypothesis is that auxin itself may oscillate. In this study, we confirm Mitchison's observation that this may indeed be the case in flux-based models. We bring new insight into the conditions under which this occurs and show that oscillations can persist when cell divisions are included in the simulations. Note that oscillatory behavior is not specific to flux-based models, as oscillations have also been observed in concentration-based models, both in simulations representing auxin distribution in roots [18] and in a numerical bifurcation study considering a row of cells [8]. Interestingly, it has been proved recently in [9] that oscillations were not possible in the limit of no auxin diffusion for the concentration-based models introduced in [13].

Although the data presented in [22] suggest that the observed oscillations do not directly involve auxin itself, but only some of its downstream targets, the present study and cited previous works show that auxin oscillations cannot be entirely ruled out. Since mathematical models indicate that auxin signaling may induce oscillations [17], in a tissue where cells divide there are potentially (at least) three oscillatory mechanisms, oscillations of auxin, oscillations of the auxin signaling pathway, and oscillations of the cell cycle, which result in cell divisions. Further investigations of these three potential tightly coupled clocks may help decipher the current conundrum of experimentally observed periodic signals in the root.

We should mention that, for a regular tissue with the structure of a ring or a row of cells, the system possesses some symmetry. However, the symmetric properties of the model have remained largely unstudied, although we have observed the antiphased oscillations in the tissue with row-like cells, which can provide insight into the dynamics of the model from the effect of the topology of coupling among cells. Are there any other kinds of oscillation waves normally exhibited in a symmetrical system, as in $[4,6,5,12]$ ? What is the relative influence of the topological structure of the tissue and the dynamics of interaction between auxin and transporter proteins on oscillations? Is it possible to characterize the nonhomogeneous patterns and their bifurcations for particular topological structures presenting symmetry? These are the subjects of further study.

Appendix A. Jacobian matrix of the three-cell ring. When $N=3$, we compute the Jacobian of (3.9)-(3.10) at the steady states of the form $E_{*}=\left(a^{*}, a^{*}, a^{*}, P_{12}, P_{23}, P_{31}\right.$, $\left.P_{13}, P_{32}, P_{21}\right)$, including $E_{0}, E_{+}$, and $E_{-}$. They can all be written in block form as

$$
J_{*}=\left[\begin{array}{c|c}
L_{*} & M \\
\hline Q_{*} & R_{*}
\end{array}\right],
$$

where the blocks marked by $*$ depend on the particular equilibrium that is considered, among $E_{0}, E_{+}$, and $E_{-}$.

The common block is

$$
M=\left[\begin{array}{rrrrrr}
-a^{*} & 0 & a^{*} & -a^{*} & 0 & a^{*} \\
a^{*} & -a^{*} & 0 & 0 & a^{*} & -a^{*} \\
0 & a^{*} & -a^{*} & a^{*} & -a^{*} & 0
\end{array}\right]
$$

Copyright $\odot$ by SIAM. Unauthorized reproduction of this article is prohibited. 
The block $L_{*}$ can be seen as a Laplacian for the oriented graph $\mathcal{G}^{+}\left(\Phi^{*}\right)$ and takes the following values, depending on each steady state:

$$
\begin{gathered}
L_{0}=\left[\begin{array}{rrr}
-(2 D+\delta) & D & D \\
D & -(2 D+\delta) & D \\
D & D & -(2 D+\delta)
\end{array}\right], \\
L_{+}=\left[\begin{array}{rrr}
-\left(2 D+\delta+P_{1}^{*}\right) & D & D+P_{1}^{*} \\
D+P_{1}^{*} & -\left(2 D+\delta+P_{1}^{*}\right) & D \\
D & D+P_{1}^{*} & -\left(2 D+\delta+P_{1}^{*}\right)
\end{array}\right],
\end{gathered}
$$

and

$$
L_{-}=\left[\begin{array}{rrr}
-\left(2 D+\delta+P_{1}^{*}\right) & D+P_{1}^{*} & D \\
D & -\left(2 D+\delta+P_{1}^{*}\right) & D+P_{1}^{*} \\
D+P_{1}^{*} & D & -\left(2 D+\delta+P_{1}^{*}\right)
\end{array}\right] \text {. }
$$

At $E_{0}=\left(a^{*}, a^{*}, a^{*}, 0,0,0,0,0,0\right)$, all flux values are of the form $\Phi_{i, i+1}^{*}=0$, and the value of $h^{\prime}(0)$ is either zero when $h$ is $C^{1}$ or discontinuous with $h^{\prime}\left(0^{+}\right)>0$ and $h^{\prime}\left(0^{-}\right)=0$. When $h^{\prime}(0)=0$, it is easy to obtain that the block matrix $Q_{0}$ is a zero matrix and $R_{0}=\mu I$, a multiplication of a scalar $\mu$ and an identity matrix. Therefore, at $E_{0}$, the Jacobian matrix

$$
J_{0}=\left[\begin{array}{rrrrrrrrr}
-(2 D+\delta) & D & D & -a^{*} & 0 & a^{*} & -a^{*} & 0 & a^{*} \\
D & -(2 D+\delta) & D & a^{*} & -a^{*} & 0 & 0 & a^{*} & -a^{*} \\
D & D & -(2 D+\delta) & 0 & a^{*} & -a^{*} & a^{*} & -a^{*} & 0 \\
0 & 0 & 0 & -\mu & 0 & 0 & 0 & 0 & 0 \\
0 & 0 & 0 & 0 & -\mu & 0 & 0 & 0 & 0 \\
0 & 0 & 0 & 0 & 0 & -\mu & 0 & 0 & 0 \\
0 & 0 & 0 & 0 & 0 & 0 & -\mu & 0 & 0 \\
0 & 0 & 0 & 0 & 0 & 0 & 0 & -\mu & 0 \\
0 & 0 & 0 & 0 & 0 & 0 & 0 & 0 & -\mu
\end{array}\right]
$$

When $h^{\prime}\left(0^{+}\right)>0$, to obtain the components in the matrices $Q_{0}$ and $R_{0}$, denote $G_{i j}=$ $h\left(\Phi_{i j}\right)-\mu P_{i j}$. From $\Phi_{i j}=\Phi_{i j}\left(a_{i}, a_{j}, P_{i j}, P_{j i}\right)=\left(P_{i j}+D\right) a_{i}-\left(P_{j i}+D\right) a_{j}$, it follows that for any $\varepsilon>0$ one has

$$
\begin{aligned}
& \Phi_{i j}\left(a_{i}+\varepsilon, a_{j}, P_{i j}, P_{j i}\right) \geqslant \Phi_{i j}\left(a_{i}, a_{j}, P_{i j}, P_{j i}\right), \\
& \Phi_{i j}\left(a_{i}, a_{j}+\varepsilon, P_{i j}, P_{j i}\right) \leqslant \Phi_{i j}\left(a_{i}, a_{j}, P_{i j}, P_{j i}\right), \\
& \Phi_{i j}\left(a_{i}, a_{j}, P_{i j}+\varepsilon, P_{j i}\right) \geqslant \Phi_{i j}\left(a_{i}, a_{j}, P_{i j}, P_{j i}\right), \\
& \Phi_{i j}\left(a_{i}, a_{j}, P_{i j}, P_{j i}+\varepsilon\right) \leqslant \Phi_{i j}\left(a_{i}, a_{j}, P_{i j}, P_{j i}\right) .
\end{aligned}
$$

Therefore, the right partial derivative

$$
\frac{\partial G_{i j}^{+}}{\partial a_{i}}\left(E_{0}\right)=\lim _{\substack{\varepsilon \rightarrow 0 \\ \varepsilon>0}} \frac{G_{i j}\left(a_{i}+\varepsilon\right)-G_{i j}\left(a_{i}\right)}{\varepsilon}=\left(P_{i j}+D\right) h^{\prime}\left(0^{+}\right)
$$

and

$$
\frac{\partial G_{i j}^{+}}{\partial a_{j}}\left(E_{0}\right)=\lim _{\substack{\varepsilon \rightarrow 0 \\ \varepsilon>0}} \frac{G_{i j}\left(a_{j}+\varepsilon\right)-G_{i j}\left(a_{i}\right)}{\varepsilon}=0 .
$$

Copyright (C) by SIAM. Unauthorized reproduction of this article is prohibited. 
Similarly,

$$
\frac{\partial G_{i j}^{+}}{\partial P_{i j}}\left(E_{0}\right)=a_{i} h^{\prime}\left(0^{ \pm}\right) \text {and } \frac{\partial G_{i j}}{\partial P_{j i}}\left(E_{0}\right)=0
$$

Hence, we have

$$
Q_{0}=\left[\begin{array}{ccc}
D h^{\prime}\left(0^{+}\right) & 0 & 0 \\
0 & D h^{\prime}\left(0^{+}\right) & 0 \\
0 & 0 & D h^{\prime}\left(0^{+}\right) \\
D h^{\prime}\left(0^{+}\right) & 0 & 0 \\
0 & 0 & D h^{\prime}\left(0^{+}\right) \\
0 & D h^{\prime}\left(0^{+}\right) & 0
\end{array}\right]
$$

and

$$
R_{0}=\left[\begin{array}{cccccc}
a^{*} h_{0}^{\prime}-\mu & 0 & 0 & 0 & 0 & 0 \\
0 & a^{*} h_{0}^{\prime}-\mu & 0 & 0 & 0 & 0 \\
0 & 0 & a^{*} h_{0}^{\prime}-\mu & 0 & 0 & 0 \\
0 & 0 & 0 & a^{*} h_{0}^{\prime}-\mu & 0 & 0 \\
0 & 0 & 0 & 0 & a^{*} h_{0}^{\prime}-\mu & 0 \\
0 & 0 & 0 & 0 & 0 & a^{*} h_{0}^{\prime}-\mu
\end{array}\right]
$$

At $E_{+}$, note that $\mathcal{P}_{1}^{*}>0$, and denote $h_{*}^{\prime}=h^{\prime}\left(a^{*} \mathcal{P}_{1}^{*}\right)$. Then, the other blocks of $J_{+}$are

$$
Q_{+}=\left[\begin{array}{rrr}
\left(D+\mathcal{P}_{1}^{*}\right) h_{*}^{\prime} & -D h_{*}^{\prime} & 0 \\
0 & \left(D+\mathcal{P}_{1}^{*}\right) h_{*}^{\prime} & -D h_{*}^{\prime} \\
-D h_{*}^{\prime} & 0 & \left(D+\mathcal{P}_{1}^{*}\right) h_{*}^{\prime} \\
0 & 0 & 0 \\
0 & 0 & 0 \\
0 & 0 & 0
\end{array}\right]
$$

and

$$
R_{+}=\left[\begin{array}{rrrrrr}
a^{*} h_{*}^{\prime}-\mu & 0 & 0 & 0 & 0 & -a^{*} h_{*}^{\prime} \\
0 & a^{*} h_{*}^{\prime}-\mu & 0 & 0 & -a^{*} h_{*}^{\prime} & 0 \\
0 & 0 & a^{*} h_{*}^{\prime}-\mu & -a^{*} h_{*}^{\prime} & 0 & 0 \\
0 & 0 & 0 & -\mu & 0 & 0 \\
0 & 0 & 0 & 0 & -\mu & 0 \\
0 & 0 & 0 & 0 & 0 & -\mu
\end{array}\right] .
$$

Similarly, we have

$$
Q_{-}=\left[\begin{array}{rrr}
0 & 0 & 0 \\
0 & 0 & 0 \\
0 & 0 & 0 \\
\left(D+\mathcal{P}_{1}^{*}\right) h_{*}^{\prime} & 0 & -D h_{*}^{\prime} \\
0 & -D h_{*}^{\prime} & \left(D+\mathcal{P}_{1}^{*}\right) h_{*}^{\prime} \\
-D h_{*}^{\prime} & \left(D+\mathcal{P}_{1}^{*}\right) h_{*}^{\prime} & 0
\end{array}\right]
$$


and

$$
R_{-}=\left[\begin{array}{rrrrrr}
-\mu & 0 & 0 & 0 & 0 & 0 \\
0 & -\mu & 0 & 0 & 0 & 0 \\
0 & 0 & -\mu & 0 & 0 & 0 \\
0 & 0 & -a^{*} h_{*}^{\prime} & a^{*} h_{*}^{\prime}-\mu & 0 & 0 \\
0 & -a^{*} h_{*}^{\prime} & 0 & 0 & a^{*} h_{*}^{\prime}-\mu & 0 \\
-a^{*} h_{*}^{\prime} & 0 & 0 & 0 & 0 & a^{*} h_{*}^{\prime}-\mu
\end{array}\right]
$$

in $J_{-}$.

\section{REFERENCES}

[1] J. Bang-Jensen And G. Gutin, Digraphs. Theory, Algorithms and Applications, 2nd ed., SpringerVerlag, New York, 2009.

[2] E. M. Bayer, R. S. Smith, T. Mandel, N. Nakayama, M. Sauer, P. Prusinkiewicz, And C. KuHLEMEIER, Integration of transport-based models for phyllotaxis and midvein formation, Genes Development, 23 (2009), pp. 373-384.

[3] A. Berman and R. J. Plemmons, Nonnegative Matrices in the Mathematical Sciences, 2nd ed., SIAM, Philadelphia, 1994.

[4] K. B. Blyuss, The effects of symmetry on the dynamics of antigenic variation, J. Math. Biol., 66 (2013), pp. $115-137$.

[5] C. O. Buckee And S. Gupta, A Network Approach to Understanding Pathogen Population Structure, Infectious Disease Informatics, Springer, New York, 2010.

[6] P.-L. Buono And V. G. LeBlanc, Realization of critical eigenvalues for scalar and symmetric linear delay-differential equations, SIAM J. Appl. Dyn. Syst., 7 (2008), pp. 1323-1354.

[7] I. De Smet, T. Tetsumura, B. De Rybel, N. F. Frey, L. Laplaze, I. Casimiro, R. Swarup, M. Naudts, S. Vanneste, D. Audenaert, D. Inzé, M. J. Bennett, and T. Beeckman, Auxindependent regulation of lateral root positioning in the basal meristem of Arabidopsis, Development, 134 (2007), pp. 681-690.

[8] D. Draelants, J. Broeckhove, G. T. S. Beemster, and W. Vanroose, Numerical bifurcation analysis of the pattern formation in a cell based auxin transport model, J. Math. Biol., 2012, DOI 10.1007/S00285-012-0588-8.

[9] C. Feller, J. P. Gabriel, C. Mazza, and F. Yerly, Pattern formation in auxin flux, J. Math. Biol., 2013, DOI 10.1007/S00285-013-0655-9.

[10] F. G. Feugier, A. Mochizuki, And Y. Iwasa, Self-organization of the vascular system in plant leaves: Inter-dependent dynamics of auxin flux and carrier proteins, J. Theoret. Biol., 236 (2005), pp. 366375 .

[11] N. Geldner, J. Friml, Y.-D. Stierhof, G. Jürgens, and K. Palme, Auxin transport inhibitors block PIN1 cycling and vesicle trafficking, Nature, 413 (2001), pp. 425-428.

[12] S. Guo And Y. Yuan, Pattern formation in a ring network with delay, Math. Models Methods Appl. Sci., 10 (2009), pp. 1797-1852.

[13] H. Jönsson, M. Heisler, B. E. Shapiro, E. M. Meyerowitz, and E. Mjolsness, An auxin-driven polarized transport model for phyllotaxis, Proc. Natl. Acad. Sci. USA, 103 (2006), pp. 1633-1638.

[14] P. Krupinski And H. Jönsson Modeling auxin-regulated development, Cold Spring Harb. Perspect. Biol., $2(2010), \mathrm{a} 001560$.

[15] R. M. H. Merks, Y. VAn de Peer, D. Inzé, and G. T. S. Beemster, Canalization without flux sensors: A traveling-wave hypothesis, TRENDS Plant Sci., 12 (2007), pp. 384-390.

[16] M. Mesbahi And M. Egerstedt, Graph Theoretic Methods in Multiagent Networks, Princeton University Press, Princeton, NJ, 2010.

[17] A. M. Middleton, J. R. King, M. J. Bennett, and M. R. Owen, Mathematical modelling of the Aux/IAA negative feedback loop, Bull. Math. Biol., 72 (2010), pp. 1383-1407.

Copyright $\odot$ by SIAM. Unauthorized reproduction of this article is prohibited. 
[18] V. V. Mironova, N. A. Omelyanchuk, G. Yosiphon, S. I. Fadeev, N. A. Kolchanov, E. MuolSNess, AND V. A. Likhoshvai, A plausible mechanism for auxin patterning along the developing root, BMC Systems Biol., 4 (2010), p. 98.

[19] G. J. Mitchison, A model for vein formation in higher plants, Proc. R. Soc. Lond. B, 207 (1980), pp. $79-109$.

[20] G. J. Mitchison, The dynamics of auxin transport, Proc. R. Soc. Lond. B, 209 (1980), pp. $489-511$.

[21] G. J. Mitchison, The polar transport of auxin and vein patterns in plants, Philos. Trans. R. Soc. Lond. B, 295 (1981), pp. 461-471.

[22] M. A. Moreno-Risueno, J. M. Van Norman, A. Moreno, J. Zhang, S. E. Ahnert, And P. N. BENFEY, Oscillating gene expression determines competence for periodic Arabidopsis root branching, Science, 329 (2010), pp. 1306-1311.

[23] P. Prusinkiewicz, S. Crawford, R. S. Smith, K. Ljung, T. Bennett, V. Ongaro, and O. Leyser, Control of bud activation by an auxin transport switch, Proc. Natl. Acad. Sci. USA, 106 (2009), pp. $17431-17436$.

[24] A. G. Rolland-Lagan and P. Prusinkiewicz, Reviewing models of auxin canalization in the context of leaf vein pattern formation in arabidopsis, Plant J., 44 (2005), pp. 854-865.

[25] P. Sahlin, B. Söderberg, And H. Jönsson, Regulated transport as a mechanism for pattern generation: Capabilities for phyllotaxis and beyond, J. Theoret. Biol., 258 (2009), pp. 60-70.

[26] R. S. Smith, S. Guyomarc'h, T. Mandel, D. Reinhardt, C. Kuhlemeier, and P. Prusinkiewicz, A plausible model of phyllotaxis, Proc. Natl. Acad. Sci. USA, 103 (2005), pp. 1301-1306.

[27] S. Stoma, M. Lucas, J. Chopard, M. Schaedel, J. Traas, and C. Godin, Flux-based transport enhancement as a plausible unifying mechanism for auxin transport in meristem development, PLoS Comput. Biol., 4 (2008), e1000207.

[28] A. M. Turing, The chemical basis of morphogenesis, Philos. Trans. R. Soc. London, 237 (1952), pp. 3772.

[29] T. Vernoux, F. Besnard, and J. TraAs, Auxin at the shoot apical meristem, Cold Spring Harb. Perspect. Biol., 2 (2010), a001487.

Copyright $@$ by SIAM. Unauthorized reproduction of this article is prohibited. 